\title{
Paramagnetically induced nuclear magnetic resonance relaxation in solutions containing $S \geqslant 1$ ions: A molecular-frame theoretical and physical model
}

Robert Sharp, Shawn M. Abernathy, and Lawrence L. Lohr

Department of Chemistry, The University of Michigan, Ann Arbor, Michigan 48109-1055

(Received 26 June 1997; accepted 14 August 1997)

\begin{abstract}
The enhancement of nuclear magnetic resonance (NMR) relaxation rates produced by paramagnetic solutes is physically rather different for electron spin $S=1 / 2$ paramagnetic species than for $S \geqslant 1$ species due to the presence of zero-field splitting interactions in the electron spin Hamiltonians of the latter. When the zfs energy is larger than the electronic Zeeman energy, the electron spin precessional motion is spatially quantized with respect to the molecule-fixed principal axis system (PAS) of the zfs tensor rather than along the external laboratory magnetic field. An analytical theory of the orthorhombic zfs limit has been derived in which the motion of the electron spin variables is described in the zfs-PAS and that of the nuclear spin variables in the laboratory coordinate frame. The resulting theoretical expressions are simple in form and suggest a physically transparent interpretation of the experiment. The NMR relaxation enhancement $R_{1 p}$ results from additive contributions, $R_{1 x}, R_{1 y}$, and $R_{1 z}$, arising from the molecular-frame Cartesian components of the time-dependent electron spin magnetic moment operator $\mu_{r}(t)$. Each Cartesian component $R_{1 r}$ depends on the dipolar power density at the nuclear Larmor frequency that is produced by the corresponding Cartesian component of $\mu_{r}(t)$. The theory displays the dependence of the relaxation enhancement on the variables of molecular structure in a very simple and physically transparent form: $R_{1 r} \propto r^{-6}\left[1+P_{2}\left(\cos \theta_{r}\right)\right]$, where $r$ is the interspin distance and $\cos \theta_{r}$ is the direction cosine of the interspin vector with the $r$ th principal axis of the zfs tensor. New experimental data are presented for the model $S=1$ complex [trans- $\left.\mathrm{Ni}(\mathrm{II})(\mathrm{acac})_{2}\left(\mathrm{H}_{2} \mathrm{O}\right)_{2}\right]$ (acac=acetylacetonato) in dioxane solvent. The magnetic field dependence of the proton $T_{1}$ of the axial water ligands has been measured over the range 0.15-1.5 T, the lower end of which corresponds to the zfs limit. The experimental data have been analyzed using the new analytical theory for the zfs-limit regime in conjunction with spin dynamics simulations in the intermediate regime. Dipolar density power plots are presented as graphical devices which clearly exhibit the physical information in the experiment, and which permit a rapid differentiation of the sensitive and insensitive parameters of theory. The data analysis depends strongly on the zfs parameter $|E|$ and on the electron spin relaxation time $\tau_{S, z}$ along the zfs-PAS $z$-axis, but only very weakly on the other parameters of theory. A fit of the data to theory provided the values $|E|=1.8 \pm 0.1 \mathrm{~cm}^{-1}$ and $\tau_{S, z}=8.0 \pm 0.3 \mathrm{ps}$. (C) 1997 American Institute of Physics. [S0021-9606(97)51643-6]
\end{abstract}

\section{INTRODUCTION}

Paramagnetic transition metal ions in solution can produce large NMR relaxation enhancements of nuclear spins on ligand species and on solvent molecules. This phenomenon, called NMR-paramagnetic relaxation enhancement or NMR-PRE, has been used widely to probe the structure, dynamics, and magnetic properties of dissolved paramagnetic species. The classical theory of the NMR-PRE was developed by Solomon, ${ }^{1}$ Bloembergen, ${ }^{2,3}$ and Morgan $^{3}$ (SBM theory), and dates from around 1961. SBM theory assumes that the nuclear and electron spins are driven by a large Zeeman interaction and undergo Larmor precession. This assumption is valid for paramagnetic species with electron spin $S=1 / 2$, but not necessarily for spin $S \geqslant 1$ transition metal ions, which are in general subject to both Zeeman and zerofield splitting (zfs) interactions. The zfs interaction involves coupling of the electron spin with the orbital angular momenta of ground and/or excited electronic states, mediated by spin-orbit coupling (the quadratic zfs interaction is equivalent to the electronic quadrupole interaction in the terminology of Abragram and Bleaney ${ }^{4}$ ). ZFS splittings may also arise from dipolar electron spin-electron spin interactions. When the zfs Hamiltonian is comparable to or greater than the Zeeman Hamiltonian $\left(H_{\mathrm{zfs}} \geqslant H_{\text {Zeem }}\right)$, zfs interactions exert a profound effect on the electron spin precessional motion. In the zfs limit $\left(H_{\mathrm{zfs}} \gg H_{\text {Zeem }}\right)$, the electron spin oscillates along the molecule-fixed axes of the zfs principal axis system (zfs-PAS), rather than precessing about the external magnetic field $B_{o}$. One of us (L.L.L.) has recently presented ${ }^{5}$ graphical displays of the semiclassical motions in such spin systems. In the intermediate regime where $H_{\mathrm{zfs}}$ $\approx H_{\text {Zeem }}$, the quantization axis undergoes a characteristic shift from molecule-fixed zfs-PAS at lower fields to the laboratory-fixed PAS of the Zeeman interaction at higher fields.

This reorientation of the quantization axis of electron spin motion has a profound influence on the NMR-PRE, and 
a great deal of theoretical effort has been expended in recent years to develop relaxation theory which is appropriate at magnetic field strengths below those of the Zeeman-limit regime. ${ }^{6-33}$ Analytical expressions which parallel the form of SBM theory but are appropriate to the uniaxial zfs limit rather than the Zeeman limit have been derived. ${ }^{19,20}$ The presence of an orthorhombic component in the zfs tensor (i.e., a nonzero zfs $E$ parameter) has a particularly large effect on the paramagnetic relaxation enhancement in the zfs limit and intermediate regimes for integer spin systems, in many cases depressing the NMR-PRE by an order of magnitude or more relative to the uniaxial $(E=0)$ situation. $^{21,24,25,34}$

While theory describing the effects of nonzero zfs interactions has advanced considerably in recent years, a simple, physically transparent model of spin relaxation in the orthorhombic zfs limit has been needed to guide interpretation, in particular to provide a clear understanding of the physical information that is present in NMR-PRE experiments. The theory developed here is suited to this purpose. New zfslimit and intermediate regime intramolecular proton $T_{1}$ data for the water ligands of the $S=1$ complex $\left[\mathrm{Ni}(\mathrm{II})(\mathrm{acac})_{2}\left(\mathrm{H}_{2} \mathrm{O}\right)_{2}\right]($ acac $=$ acetylacetonato $)$ are presented. It is shown that the use of spectral density profiles to interpret the zfs-limit behavior, in conjunction with spin dynamics simulations to describe behavior in the intermediate regime, provides an accurate and general computational platform within a framework which is highly intuitive physically. Spectral density profiles clearly display the information content of the NMR-PRE experiment and differentiate between the sensitive and insensitive parameters of theory.

An additional advantage of the molecular frame theoretical formulation described below is that it provides a straightforward way of incorporating anisotropic molecular frame tensors into the theory. The most important kind of molecular anisotropy is probably that of electron spin relaxation; i.e., the electron spin relaxation rate differs along different Cartesian axes of the molecular coordinate system. While Zeeman-limit (SBM) theory has been modified ${ }^{35}$ to incorporate separate laboratory-frame longitudinal and transverse electron spin relaxation times, $\tau_{S 1}$ and $\tau_{S 2}$, the molecular-frame anisotropy of electron spin relaxation has not previously been addressed. Such anisotropy probably has important experimental consequences when the electronic anisotropy of the metal ion is large. A very interesting example is the water-soluble $\mathrm{Mn}(\mathrm{III})$ porphyrin, tetraphenylsulfonato-Mn(III) porphyrin (Mn(III)-TTPS 4 ), which has been studied extensively ${ }^{36-38}$ in regard to its potential use as an MRI contrast agent. The $T_{1}$ water proton relaxivity of this complex is highly unusual, both in its exceptionally large magnitude [which is higher, per bound water molecule, than that of hexaaqua-Mn(II)], as well as in its very unusual magnetic field dependence. The extremely high inner sphere relaxivity, which is unparalleled among known $\mathrm{Mn}(\mathrm{III})$ complexes, probably (in our view) results from an anomalously long electron spin relaxation time along the fourfold axis of the porphyrin. Molecular frame descriptions of both electron and nuclear spin relaxation are needed. The theory developed below provides the latter, i.e., a molecularframe description of nuclear spin relaxation which can accommodate anisotropic electron spin relaxation parameters. A theory of anisotropic electron spin relaxation in the molecular coordinate frame will be published in a subsequent study.

\section{NMR-PRE IN THE ORTHORHOMBIC ZFS LIMIT}

Our objectives are twofold: first to recast the theoretical expressions used in the spin dynamics algorithms of SpinDyn. $\mathrm{f}^{33}$ into a molecular coordinate frame description which is suitable for incorporating anisotropic electron spin relaxation and anisotropic molecular reorientation parameters. Second, it will also be shown that the molecular-frame description developed below leads to a highly transparent physical interpretation of paramagnetic relaxation enhancement in the orthorhombic zfs limit.

From the linear response theory of Kubo and Tomita, ${ }^{39,40}$ the $T_{1}$ and $T_{2}$ NMR relaxation rates $\left[\equiv R_{1(2) p}\right]$ are given by,

$$
R_{1(2) p}=\left(\hbar^{2}\left\langle I_{q}^{2}\right\rangle\right)^{-1} \int_{0}^{\infty}\left\langle\left[I_{q}, H_{S}^{\prime}(t)\right]\left[H_{S}^{\prime}(0), I_{q}\right]\right\rangle d t,
$$

where $q=z(x)$ for $T_{1(2)} . H_{S}^{\prime}(t)$ is the time-dependent Hamiltonian which couples the electron $(S)$ and nuclear $(I)$ spins. The angular brackets around the product of commutators in the integrand denote expectation values of the spin variables and an ensemble average over the spatial variables of $H_{S}^{\prime}$. We consider only the magnetic dipole-magnetic dipole contribution to relaxation (neglecting the scalar hyperfine interaction), in which case $H_{S}^{\prime}$ can be written

$$
H_{S}^{\prime}=\frac{\hbar \kappa}{r^{3}} \sum_{q} 3^{-1 / 2}(-1)^{1-q} I_{q}^{(1)} F_{q}^{(1)}
$$

where

$$
\begin{aligned}
& \kappa=-30^{1 / 2} \gamma_{I} g \beta_{o}\left(\mu_{o} / 4 \pi\right), \\
& I_{ \pm}^{(1)}=\mp 2^{-1 / 2} I_{ \pm}, \\
& I_{o}^{(1)}=I_{z}, \\
& F_{m}^{(1)}=\left(\frac{4 \pi}{5}\right)^{1 / 2} \sum_{p=-1}^{1} c_{m, p} S_{p}^{(1)} Y_{m-p}^{(2)}(\theta, \varphi) .
\end{aligned}
$$

$\gamma_{I}$ is the nuclear gyromagnetic ratio, $g$ is the electron $g$-factor, $\beta_{o}$ is the Bohr magneton, and $\mu_{o}$ is the magnetic permeability of free space. $S_{p}^{(1)}$ is components of the electron spin vector, written in spherical tensor form analogous to Eqs. (4). $Y_{q}^{(2)}(\theta, \varphi)$ are spherical harmonics of the polar variables $(\theta, \varphi)$ which specify the orientation of the interspin vector $\bar{r}_{\mathrm{IS}}$ in the laboratory coordinate frame. The coefficients $c_{m, p}$ are 22

$$
\begin{aligned}
& c_{+1,+1}=c_{-1,-1}=10^{-1 / 2}, \\
& c_{+1,0}=c_{-1,0}=-(3 / 10)^{1 / 2}, \\
& c_{0,+1}=c_{0,-1}=(3 / 10)^{1 / 2},
\end{aligned}
$$




$$
\begin{aligned}
& c_{+1,-1}=c_{-1,+1}=(3 / 5)^{1 / 2}, \\
& c_{0,0}=-(2 / 5)^{1 / 2} .
\end{aligned}
$$

Evaluation of the commutators in Eq. (1) gives the following expression for $R_{1 p}$ :

$$
\begin{aligned}
R_{1 p}= & -\left(\frac{3^{-1 / 2} \kappa}{r^{3}}\right)^{2} \int_{0}^{\infty}\left\{\left\langle F_{+1}^{(1)}(t) F_{-1}^{(1)}(0)\right\rangle e^{-i \omega_{I} t}\right. \\
& + \text { c.c. }\}_{\text {en av }} d t .
\end{aligned}
$$

\section{THE ORTHORHOMBIC ZFS LIMIT}

Equations (1), (2), and (4)-(6) are all expressed in the laboratory coordinate frame. Our objective is to develop a formulation suitable for describing electron spin motion in the vicinity of the zfs limit, where it is quantized along the axes of the zfs-PAS. For this purpose, the lattice tensor $F^{(1)}$ is expressed in the molecule-fixed coordinate frame,

$$
F_{m}^{(1)}=\sum_{q=-1}^{+1} \hat{F}_{q}^{(1)} D_{q, m}^{(1)}(\alpha \beta \gamma),
$$

where the $D_{q, m}^{(1)}(\alpha \beta \gamma)$ are Wigner rotation matrix elements and $\alpha, \beta, \gamma$ are the Euler angles which rotate the laboratory coordinate frame to the molecule-fixed coordinate frame. This gives

$$
\begin{aligned}
\left\langle F_{+1}^{(1)}(t) F_{-1}^{(1)}(0)\right\rangle & \\
= & \left(\frac{4 \pi}{5}\right) \sum_{q, q^{\prime}}\left\{D_{q,+1}^{(1)}(\alpha \beta \gamma ; t) D_{q^{\prime},-1}^{(1)}(\alpha \beta \gamma ; 0)\right. \\
& \times \sum_{p, p^{\prime}} c_{q, p} c_{q^{\prime}, p^{\prime}}, \hat{Y}_{q-p}^{(2)}(\theta, \varphi) \\
& \left.\times \hat{Y}_{q^{\prime}-p^{\prime}}^{(2)}(\theta, \varphi) \operatorname{Tr}\left[\rho_{S} \hat{S}_{p}^{(1)}(t) \hat{S}_{p^{\prime}}^{(1)}(0)\right]\right\}_{\mathrm{ea}},
\end{aligned}
$$

where $\rho_{S}$ is the density matrix of the $S$ spin system, and the curly brackets denote an ensemble average over the spatial variables. We use the convention that quantities with superscripting carets $\left({ }^{\wedge}\right)$ are defined in the molecular coordinate frame, while the corresponding quantities without carets are defined in the laboratory coordinate frame.

We now particularize Eq. (8) to the orthorhombic zfs limit $\left(D, E \neq 0, B_{o}=0\right)$ when molecular reorientation is slow, in which case Eq. (8) can be written in closed form. Slow reorientational motion implies, in the context of Eq. (8), that the time scale of electron spin relaxation is rapid compared to that of Brownian reorientation, in which case $D_{q, r}^{(1)}(\alpha \beta \gamma ; t) \cong D_{q, r}^{(1)}(\alpha \beta \gamma ; 0)$. In isotropic media, the spatial averages of the Wigner rotation matrix elements are given by

$$
\overline{D_{q, r}^{(1)}(\alpha \beta \gamma) D_{q^{\prime}, r^{\prime}}^{(1)}(\alpha \beta \gamma)}=3^{-1} \delta_{q^{\prime},-q} \delta_{r^{\prime},-r} .
$$

This relation requires that $q^{\prime}=-q$ in Eq. (8).
To evaluate the trace over the variables of $S$, we assume that the electron spin system remains at thermal equilibrium during the relaxation of $I$, which is nearly always the case experimentally, so that $\rho_{S}$ is diagonal,

$$
\begin{aligned}
& \rho_{S}=\sum_{\mu} P_{\mu}^{\circ}|\mu\rangle\langle\mu|, \\
& P_{\mu}^{\circ}=Q^{-1} \exp \left(-\epsilon_{\mu} / k T\right),
\end{aligned}
$$

where $|\mu\rangle$ and $\epsilon_{\mu}$ are the eigenvectors and eigenvalues of $H_{\mathrm{zfs}}, Q$ is the partition function, and $P_{\mu}^{0}$ is the thermal equilibrium population of $|\mu\rangle$. The trace can be evaluated in closed form in the eigenbasis of $H_{S}$, the stationary part of the electron spin Hamiltonian:

$$
\begin{aligned}
\operatorname{Tr}\left[\rho_{S} \hat{S}_{p}^{(1)}(t) \hat{S}_{p^{\prime}}^{(1)}(0)\right]= & \sum_{\mu, \nu} P_{\mu}^{\circ}\left\langle\mu\left|\exp \left(i \hbar^{-1} H_{S} t\right) \hat{S}_{p}^{(1)}\right| \nu\right\rangle \\
& \times\left\langle\nu\left|\exp \left(i \hbar^{-1} H_{S} t\right) \hat{S}_{p^{\prime}}^{(1)}\right| \mu\right\rangle \\
= & \sum_{\mu} P_{\mu}^{\circ} \exp \left(i\left[\omega_{\mu}-\omega_{\nu}\right] t\right) \\
& \times \exp \left(-t / \tau_{S}\right)\left\langle\mu\left|\hat{S}_{p}^{(1)}\right| \nu\right\rangle\left\langle\nu\left|\hat{S}_{p^{\prime}}^{(1)}\right| \mu\right\rangle,
\end{aligned}
$$

where $\omega_{\mu}=\hbar^{-1} \epsilon_{\mu}$, and $\tau_{S}$ is a phenomenological electron spin relaxation time.

These expressions can be put into a simple and physically transparent form in the orthorhombic zfs limit. In this case, the eigenvectors $|\mu\rangle$ transform as irreducible representations of the point group $D_{2 h}$ of the zfs tensor, and the matrix elements of $H_{\mathrm{zfs}}$ are most readily evaluated in the Cartesian basis. For integer spins $S=1$ and $S=2$, the eigenfunctions can be classified as follows:

(i) $S=1$ is spanned by three eigenfunctions of symmetry types, $B_{1 g}, B_{2 g}$, and $B_{3 g}$. These functions transform as the rotations $R_{\hat{z}}, R_{\hat{y}}$, and $R_{\hat{x}}$, respectively, and will be labeled $|\hat{z}\rangle,|\hat{y}\rangle$, and $|\hat{x}\rangle$.

(ii) $S=2$ is spanned by five eigenfunctions of symmetry types, $2 A_{g}, B_{1 g}, B_{2 g}$, and $B_{3 g}$, which transform as the Cartesian functions $\left|\hat{z}^{2}\right\rangle,\left|\hat{x}^{2}-\hat{y}^{2}\right\rangle,|\hat{x} \hat{y}\rangle,|\hat{x} \hat{z}\rangle$, and $|\hat{y} \hat{z}\rangle$, respectively. The two $A_{g}$ functions, $A_{g}^{(a)}$ and $A_{g}^{(\mathrm{b})}$, mix under $H_{\mathrm{zfs}}$ when $|E|>0$.

The angular momentum operators $\left(S_{\hat{x}}, S_{\hat{y}}, S_{\hat{z}}\right)$ transform as $B_{3 g}, B_{2 g}$, and $B_{1 g}$, respectively (i.e., like $R_{\hat{x}}, R_{\hat{y}}, R_{\hat{z}}$ ). It can readily be verified that in the Cartesian representation the matrix elements $\left\langle\mu\left|S_{\hat{r}}\right| \nu\right\rangle(\hat{r}=\hat{x}, \hat{y}, \hat{z})$ vanish if the symmetry reps of any two of the three quantities, $|\mu\rangle,|\nu\rangle$, or $S_{\hat{r}}$, are the same. For $S=1$, the only nonvanishing matrix elements are:

$$
\left|\left\langle\hat{x}\left|S_{\hat{y}}\right| \hat{z}\right\rangle\right|=\left|\left\langle\hat{y}\left|S_{\bar{x}}\right| \hat{z}\right\rangle\right|=\left|\left\langle\hat{x}\left|S_{\hat{z}}\right| \hat{y}\right\rangle\right|=1,
$$

and for $S=2$ :

$$
\begin{aligned}
& \left\langle A_{g}\left|S_{\hat{x}}\right| B_{3 g}\right\rangle, \quad\left\langle A_{g}\left|S_{\hat{y}}\right| B_{2 g}\right\rangle, \quad\left\langle A_{g}\left|S_{\hat{z}}\right| B_{1 g}\right\rangle, \\
& \left\langle B_{1 g}\left|S_{\hat{x}}\right| B_{2 g}\right\rangle, \quad\left\langle B_{1 g}\left|S_{\hat{y}}\right| B_{3 g}\right\rangle, \quad\left\langle B_{2 g}\left|S_{\hat{z}}\right| B_{3 g}\right\rangle,
\end{aligned}
$$


Using these results and Eq. (10), the trace in Eq. (8) can be evaluated directly by rewriting the spherical tensor operators $\hat{S}_{p}, \hat{S}_{p^{\prime}}$ as Cartesian operators $S_{\hat{x}}, S_{\hat{y}}, S_{\hat{z}}$. From the selection rule for $\left\langle\mu\left|S_{\hat{r}}\right| \nu\right\rangle$, it follows that cross terms such as $\left\langle\mu\left|\hat{S}_{ \pm 1}\right| \nu\right\rangle\left\langle\nu\left|\hat{S}_{0}\right| \mu\right\rangle$ and $\left\langle\mu\left|\hat{S}_{0}\right| \nu\right\rangle\left\langle\nu\left|\hat{S}_{ \pm 1}\right| \mu\right\rangle$ vanish, as do cross terms containing products such as $\left\langle\mu\left|S_{\hat{x}}\right| \nu\right\rangle\left\langle\nu\left|S_{\hat{y}}\right| \mu\right\rangle$. The only nonvanishing products of matrix elements are the following:

$$
\begin{aligned}
p= \pm 1, p^{\prime}= \pm 1: \quad\left\langle\mu\left|\hat{S}_{ \pm 1}^{(1)}\right| \nu\right\rangle\left\langle\nu\left|\hat{S}_{ \pm 1}^{(1)}\right| \mu\right\rangle= & \left|\left\langle\mu\left|S_{\hat{x}}\right| \nu\right\rangle\right|^{2} \\
& -\left|\left\langle\mu\left|S_{\hat{y}}\right| \nu\right\rangle\right|^{2},
\end{aligned}
$$

$$
\begin{aligned}
p= \pm 1, p^{\prime}=\mp 1: \quad\left\langle\mu\left|\hat{S}_{ \pm 1}^{(1)}\right| \nu\right\rangle\left\langle\nu\left|\hat{S}_{+1}^{(1)}\right| \mu\right\rangle= & \left|\left\langle\mu\left|S_{\hat{x}}\right| \nu\right\rangle\right|^{2} \\
& +\left|\left\langle\mu\left|S_{\hat{y}}\right| \nu\right\rangle\right|^{2},
\end{aligned}
$$

$p=0 ; p^{\prime}=0: \quad\left\langle\mu\left|\hat{S}_{0}^{(1)}\right| \nu\right\rangle\left\langle\nu\left|\hat{S}_{0}^{(1)}\right| \mu\right\rangle=\left|\left\langle\mu\left|S_{\hat{z}}\right| \nu\right\rangle\right|^{2}$.

Combining the results of Eqs. (8)-(13), the zfs limit $R_{1 p}$ can be expressed in the form

$$
\begin{aligned}
R_{1 p}= & \left(\frac{2 \kappa^{2}}{45 r^{6}}\right) \operatorname{Re}\left\{\int _ { 0 } ^ { \infty } \left[A G_{\hat{z}}(t)+(B+C) G_{\hat{x}}(t)\right.\right. \\
& \left.\left.+(B-C) G_{\hat{y}}(t)\right] e^{i \omega_{I} t} d t\right\},
\end{aligned}
$$

where

$$
\begin{aligned}
& A=(2 \pi / 5)\left[4 \hat{Y}_{0}^{(2)} \hat{Y}_{0}^{(2)}-6 \hat{Y}_{-1}^{(2)} \hat{Y}_{+1}^{(2)}\right], \\
& B=(2 \pi / 5)\left[+\hat{Y}_{0}^{(2)} \hat{Y}_{0}^{(2)}-3 \hat{Y}_{-1}^{(2)} \hat{Y}_{+1}^{(2)}+6 \hat{Y}_{-2}^{(2)} \hat{Y}_{+2}^{(2)}\right], \\
& C=(2 \pi / 5) \operatorname{Re}\left\{3 \hat{Y}_{-1}^{(2)} \hat{Y}_{-1}^{(2)}-2 \cdot 6^{1 / 2} \hat{Y}_{-2}^{(2)} \hat{Y}_{0}^{(2)}\right\} .
\end{aligned}
$$

The angular variables $(\theta, \varphi)$ of the spherical harmonics, which define the orientation of the interspin vector in the zfs-PAS, have been omitted for brevity. The angular functions $A, B+C$, and $B-C$ each have a simple physical interpretation which is discussed further below. The functions $G_{\hat{r}}(t)$ are time correlation functions of the electron spin component operators, defined as

$$
G_{\hat{x}}(t)=\sum_{\mu, \nu} P_{\mu}^{\circ} \exp \left(\left[i \omega_{\mu \nu}-\tau_{S, \hat{x}}^{-1}\right] t\right)\left|\left\langle\mu\left|S_{\hat{x}}\right| \nu\right\rangle\right|^{2},
$$

with analogous expressions for $G_{\hat{y}}(t)$ and $G_{\hat{z}}(t) . G_{\hat{r}}(t)$ contains one nonzero term for each spin transition $(\mu \leftrightarrow \nu)$ for which the matrix element $\left\langle\mu\left|S_{\hat{r}}\right| \nu\right\rangle$ is nonvanishing. $\tau_{S, \hat{r}}$ is a phenomenological electron spin relaxation time describing relaxation along the $\hat{\mathbf{r}}$ th molecular axis. In the orthorhombic zfs-limit, the molecular coordinate axes are physically distinct, and each is associated with a specific electron spin relaxation time $\tau_{S, \hat{r}}$.

The function $A$ in Eq. (15a) can be written in a simpler form as

$$
A=1+P_{2}\left(\cos \theta_{\hat{z}}\right),
$$

where $P_{2}\left(\cos \theta_{z}\right)$ is a second order Legendre polynomial and $\theta_{\hat{z}}$ is the polar angle of the $I-S$ interspin vector with respect

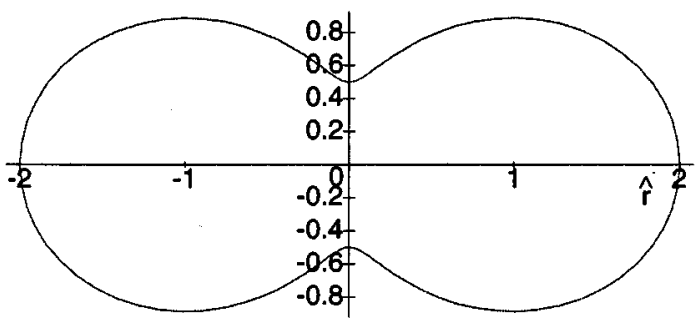

FIG. 1. Plot of the function $1+P_{2}\left(\cos \theta_{\hat{z}}\right)$.

to the $\hat{z}$ axis of the molecule-fixed zfs-PAS. Physically, the labeling of the $\hat{z}$-axis is not unique. Although by convention $\hat{z}$ is usually chosen as the principal axis for which the absolute magnitude of the zfs energy is largest, either of the other principal axes could be labeled $\hat{z}$ and the problem solved in an analogous fashion, but with different values for $D$ and $E$. (The conventional choice of $\hat{z}$-axis ensures that the $|E / D|$ ratio lies in the range 0 to $1 / 3$.) From this reasoning we infer that the coefficients of $G_{\hat{x}}(t)$ and $G_{\hat{y}}(t)$ in Eq. (14) have analogous functional forms, i.e., that

$$
\begin{aligned}
& B+C=1+P_{2}\left(\cos \theta_{\hat{x}}\right), \\
& B-C=1+P_{2}\left(\cos \theta_{\hat{y}}\right),
\end{aligned}
$$

where $\cos \theta_{\hat{x}(\hat{y})}$ are the direction cosines of the $I-S$ vector with respect to the $\hat{x}(\hat{y})$ axes. Although the mathematical relationship underlying Eqs. (17b) and (17c) is not obvious, these relations are readily confirmed by numerical calculation.

\section{A PHYSICAL MODEL OF PARAMAGNETICALLY INDUCED NMR RELAXATION IN THE ZFS LIMIT}

Summarizing the discussion of the foregoing section, it has been shown that $R_{1 p}$ can be written as a sum of contributions due, respectively, to the Cartesian components $\mu_{\hat{r}}$ $(\hat{r}=\hat{x}, \hat{y}, \hat{z})$ of $\bar{\mu}_{S}$, i.e.,

$$
\begin{aligned}
R_{1 p}= & R_{1 \hat{z}}+R_{1 \hat{x}}+R_{1 \hat{y}}, \\
R_{1 \hat{r}}= & \left(\frac{2 \kappa^{2}}{45 r^{6}}\right)\left[1+P_{2}\left(\cos \theta_{\hat{r}}\right)\right] \sum_{\mu, \nu} P_{\mu}^{\circ}\left|\left\langle\mu\left|S_{\hat{r}}\right| \nu\right\rangle\right|^{2} \\
& \times J_{\hat{r}}\left(\omega_{\mu \nu}+\omega_{I}\right), \\
J_{\hat{r}}(\omega) & =\frac{\tau_{S, \hat{r}}}{1+\omega^{2} \tau_{S, \hat{r}}^{2}} .
\end{aligned}
$$

Each contribution $R_{1 \hat{r}}$ has an angular dependence given by the function $\left[1+P_{2}\left(\cos \theta_{\hat{r}}\right)\right]$ shown in Fig. 1, where $\theta_{\hat{r}}$ is the polar angle of the $I-S$ interspin vector with respect to the $\hat{\mathbf{r}}$ th Cartesian coordinate axis. The spectral density functions $J_{\hat{r}}(\omega)$ describe the dipolar power density produced by Cartesian components $\mu_{\hat{r}}(t)$ and arise from the integrals of the time correlation functions in Eq. (14). Each Cartesian component of $R_{1 p}$ contains spectral contributions from the transitions $(|\mu\rangle \leftrightarrow|\nu\rangle)$ for which the transition matrix elements $\left\langle\mu \mid S_{\hat{r} \mid} \nu\right\rangle$ are nonzero. It should be noted that two terms, those for $\left( \pm \omega_{\mu \nu}\right)$, contribute to the sums in Eqs. (18b) for 


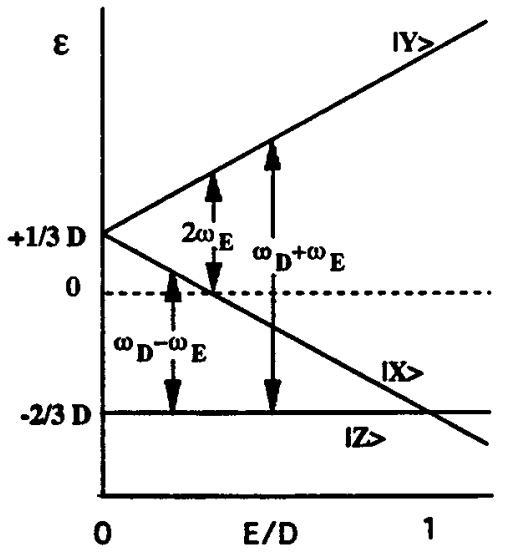

FIG. 2. Energy level diagram for a spin $S=1$ in the orthorhombic zfs limit.

each distinct electronic transition frequency $\left|\omega_{\mu \nu}\right|$. In the high temperature limit where $P_{\mu}^{\circ}=(2 S+1)^{-1}$, if we assume $\omega_{\mu \nu} \gg \omega_{I}$, these terms can be combined, giving a sum over distinct electronic transitions,

$$
\begin{aligned}
R_{1 \hat{r}}= & \left(\frac{4 \kappa^{2}}{45 r^{6}}\right)(2 S+1)^{-1}\left[1+P_{2}\left(\cos \theta_{\hat{r}}\right)\right] \\
& \times \sum_{\mu} \sum_{\nu>\mu}\left|\left\langle\mu\left|S_{\hat{r}}\right| \nu\right\rangle\right|^{2} J_{\hat{r}}\left(\omega_{\mu \nu}\right) .
\end{aligned}
$$

The geometric functions $\left[1+P_{2}\left(\cos \theta_{r}\right)\right]$ have a simple physical interpretation in that each describes the angular variation of the squared local dipolar magnetic field of a magnetic dipole $\mu_{\hat{r}}$ aligned along the $\hat{\mathbf{r}}$ th Cartesian axis. This can be shown as follows. The local dipolar field produced at a field point $\overline{\mathbf{r}}$ by a magnetic dipole of magnitude $\mu_{z}$ parallel to the $z$-axis is

$$
\bar{B}^{\prime}(\vec{r})=c_{\mu} r^{-3}(3(\hat{k} \cdot \hat{r}) \hat{r}-\hat{k}),
$$

where $\hat{\mathbf{r}}$ and $\hat{k}$ are unit vectors along $\overline{\mathbf{r}}$ and $z, c_{\mu}$ $=\left(\mu_{o} / 4 \pi\right) \mu_{z}$, and $\mu_{o}$ is the magnetic permeability of free space. The components of $\bar{B}^{\prime}(\bar{r})$ are

$$
\begin{aligned}
& B_{x}^{\prime}=c_{\mu} r^{-3}\left(3 x z / r^{2}\right)=c_{\mu} r^{-3} 3\left(\frac{2 \pi}{15}\right)^{1 / 2}\left(-Y_{+1}^{(2)}+Y_{-1}^{(2)}\right), \\
& B_{y}^{\ell}=c_{\mu} r^{-3}\left(3 y z / r^{2}\right)=c_{\mu} r^{-3}(3 i)\left(\frac{2 \pi}{15}\right)^{1 / 2}\left(Y_{+1}^{(2)}+Y_{-1}^{(2)}\right), \\
& B_{z}^{\ell}=c_{\mu} r^{-3}\left(3(z / r)^{2}-1\right)=c_{\mu} r^{-3} 4\left(\frac{\pi}{5}\right)^{1 / 2} Y_{0}^{(2)} .
\end{aligned}
$$

The squared dipolar field due to $\mu_{z}$ is then

$$
\begin{aligned}
\left|\bar{B}^{\prime}\right|^{2} & =c_{\mu}^{2} r^{-6}\left(\frac{4 \pi}{5}\right)\left(4 Y_{0}^{(2)} Y_{0}^{(2)}-6 Y_{+1}^{(2)} Y_{-1}^{(2)}\right) \\
& =2 c_{\mu}^{2} r^{-6}\left[1+P_{2}\left(\cos \theta_{z}\right)\right] .
\end{aligned}
$$

According to Eqs. (21), nuclear spin relaxation results additively from the mean-square local dipolar magnetic
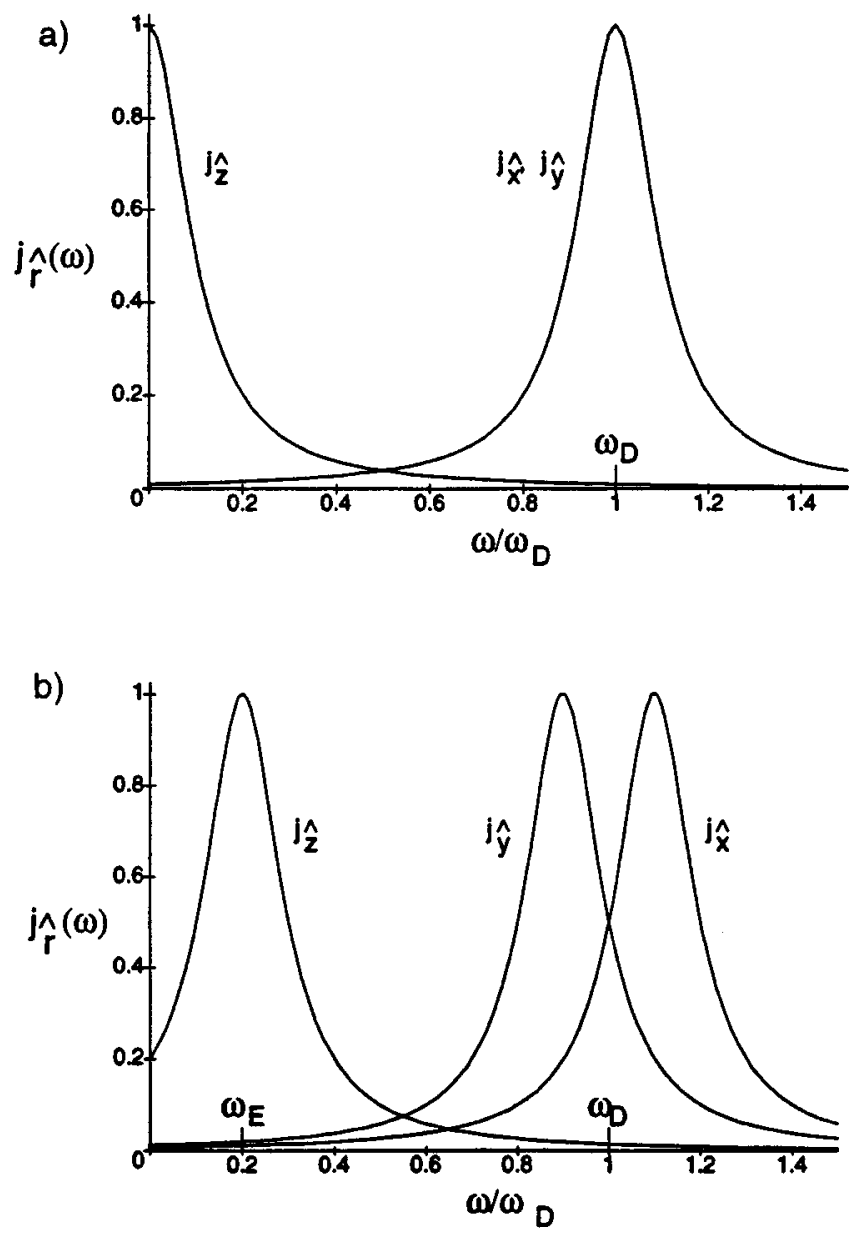

FIG. 3. Dipolar spectral density functions for $S=1$ in the uniaxial (a) and orthorhombic (b) zfs limits. The electron spin relaxation time is $\tau_{S, \hat{r}}$ $=10 \omega_{D}^{-1}$. In (a), the power spectra of $j_{\hat{x}}$ and $j_{\hat{y}}$ coincide and appear as a single band. In (b), the zfs rhombicity is $|E / D|=0.1$.

fields associated with the three Cartesian components of the electron spin magnetic moment. The field of each component operator $\mu_{\hat{r}}(t)$ oscillates coherently at the eigenfrequencies of the spin system for which the transition matrix elements $\left\langle\mu\left|S_{\hat{r}}\right| \nu\right\rangle$ are nonvanishing. For $S=1$, the matrix elements of each component operator oscillate at a single frequency: $\mu_{\hat{z}}(t)$ oscillates at $2 \omega_{E}, \mu_{\hat{x}}(t)$ at $\omega_{D}+\omega_{E}$, and $\mu_{\hat{y}}(t)$ at $\omega_{D}-\omega_{E}$, as shown in the energy level diagram of Fig. 2. Thus for $S=1, R_{1 p}$ can be written

$$
\begin{aligned}
R_{1 p}= & \left(\frac{4 \kappa^{2}}{45 r^{6}}\right) 3^{-1}\left\{\left[1+P_{2} \cos \left(\theta_{\hat{z}}\right)\right] J\left(2 \omega_{E}\right)\right. \\
& +\left[1+P_{2} \cos \left(\theta_{\hat{x}}\right)\right] J\left(\omega_{D}+\omega_{E}\right) \\
& \left.+\left[1+P_{2}\left(\cos \theta_{\hat{y}}\right)\right] J\left(\omega_{D}-\omega_{E}\right)\right\} .
\end{aligned}
$$

\section{THE DIPOLAR POWER SPECTRUM}

The dipolar power spectrum can be visualized through the Lorentzian spectral density function, $J\left(\omega_{\mu \nu}-\omega\right)$ $\equiv j\left(\omega_{\mu \nu}\right) . R_{1 p}$ is proportional to the value of $J$ at $\omega=$ $-\omega_{I}$. Figure 3 shows typical power spectra of the $I-S$ di- 
polar interaction for $S=1$ in the uniaxial [E=0, Fig. 3(a)] and orthorhombic $[E \neq 0$, Fig. 3(b)] zfs limits, respectively. Each spectrum is comprised of three Cartesian spectral density functions, each Lorentzian in shape [Eq. (19)], and each centered on the associated oscillation frequency, $\omega_{r}$, of the component $\mu_{\hat{r}}(t)$. Each power band has a width $2\left(\tau_{c, \hat{r}}\right)^{-1}$, where $\tau_{c, \hat{r}}$ is a correlation time characterizing the stochastic fluctuations in $H_{S}^{\prime}(t)$. The discussion of the previous section assumed slow Brownian reorientation, in which case $\tau_{c, \hat{r}}$ $=\tau_{S, \hat{r}}$, but in general $\left(\tau_{c, \hat{r}}\right)^{-1}$ will contain contributions from both electron spin relaxation and molecular reorientation. It is possible to account roughly for the effects of Brownian reorientation within the "decomposition approximation" by replacing $\tau_{S, \hat{r}}$ in Eq. (19) with a dipolar correlation time of the form $\tau_{c, \hat{r}}=1 /\left(\tau_{S, \hat{r}}^{-1}+\tau_{R, \hat{r}}^{-1}\right)$.

$T_{1}$ relaxation of the nuclear $(I)$ spin results from the dipolar power density at $\omega_{I}$, which is effectively zero on the scale of the electron spin transition frequencies in Fig. 3. In the uniaxial zfs limit, the frequency associated with $\mu_{\hat{z}}(t)$ approaches zero while the frequencies associated with $\mu_{\hat{x}}(t)$ and $\mu_{\hat{y}}(t)$ approach $\omega_{D}$ (Fig. 2). In this situation the contribution to $R_{1 p}$ from $R_{1 \hat{z}}$ is usually much greater than the contributions due to $R_{1 \hat{x}}$ and $R_{1 \hat{y}}$, except when $\tau_{S, \hat{r}}$ is so short that $\omega_{Y Z} \tau_{S, \hat{x}}<1$ or $\omega_{X Z} \tau_{S, \hat{y}}<1$. The dependence of $R_{1 p}$ on the nuclear position in the zfs-PAS is given by Eqs. (20), as described above.

Figure 3(b) shows the dipolar power spectrum for an orthorhombic zfs tensor with $E / D=0.1,\left(\tau_{S, \hat{r}}\right)^{-1}=0.1 \omega_{D}$. The dipolar power band associated with $\mu_{\hat{z}}(t)$ is displaced from $\omega=0$ to a center frequency of $\omega_{X Y}=2 \omega_{E}$, which causes a drop in the dipolar power available at $\omega_{I}$; when $2 \omega_{X Y} \tau_{S, \hat{z}}>1$, increasing zfs rhombicity thus produces a rapid drop in $R_{1 \hat{z}}$. The power bands associated with $\mu_{\hat{x}}(t)$ and $\mu_{\hat{y}}(t)$ are displaced, respectively, to frequencies of $\omega_{Y Z}=\omega_{D}+\omega_{E}$ and $\omega_{X Z}=\omega_{D}-\omega_{E}$. However, these high frequency bands contribute to $R_{1 p}$ significantly only when they produce significant dipolar power at $\omega_{I}$, which requires a sufficiently short $\tau_{c, \hat{r}}$ that the half width, $\left(\tau_{c, \hat{r}}\right)^{-1}$, of the power band not be too much smaller than the transition frequency, i.e., $\omega_{D}+\omega_{E}$ for $\mu_{\hat{x}}(t)$ or $\omega_{D}-\omega_{E}$ for $\mu_{\hat{y}}(t)$. However, the relative contributions of $R_{1 \hat{x}}$ and $R_{1 \hat{y}}$ to $R_{1 p}$ also depend on the orientation of the interspin vector in the molecular coordinate frame. At constant interspin distance, the high frequency bands have a fourfold greater effect at equatorial locations than at axial locations as described by Eqs. (20).

The dipolar power spectrum provides an intuitive framework for understanding relaxation phenomena in the vicinity of the zfs limit. As an example, we consider the transition from the orthorhombic zfs limit at low field to the Zeeman limit at high field. As described above, zfs rhombicity acts to suppress the NMR-PRE in the zfs limit by shifting the dipolar power band due to the $\mu_{\hat{z}}(t)$ away from zero frequency. Physically, this effect arises from the oscillation that is induced in $\mu_{\hat{z}}(t)$ by zfs rhombicity, which thereby acts to decouple the nuclear and electron spins. In the Zeeman limit, $\mu_{z}$ is static with respect to precession, and thus produces

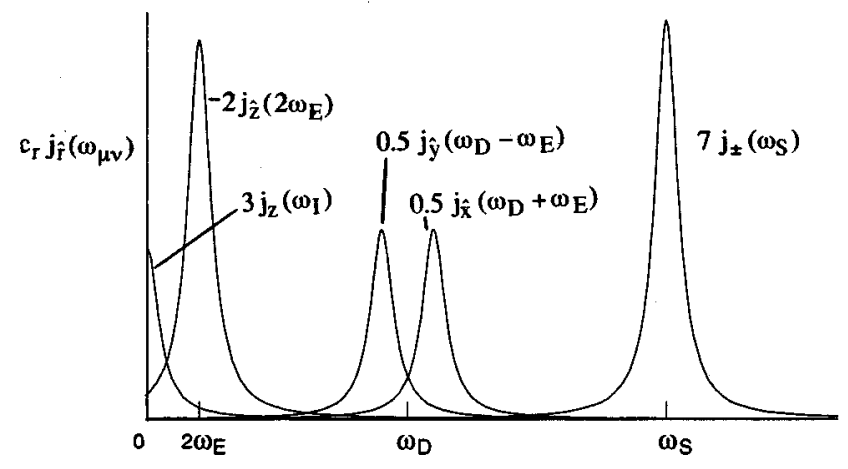

FIG. 4. Comparison of dipolar power spectra in the Zeeman and orthorhombic zfs limits. The zfs-limit spectrum $\left[2 j_{\hat{z}}\left(2 \omega_{E}\right)+0.5 j_{\hat{x}}\left(\omega_{D}+\omega_{E}\right)\right.$ $\left.+0.5 j_{\hat{y}}\left(\omega_{D}-\omega_{E}\right)\right]$ is calculated assuming $\theta=0, D=1 \mathrm{~cm}^{-1}, E / D=0.1$, $\tau_{c, \hat{r}}=10^{-10} \mathrm{~s}$. Zeeman-limit spectrum $\left[3 j_{z}\left(\omega_{I}\right)+7 j_{ \pm}\left(\omega_{S}\right)\right]$ assumes $\omega_{S}$ $=2 \omega_{D}, \tau_{c, r}=10^{-10} \mathrm{~s}$. The zfs and Zeeman spectra were multiplied by the numerical factors (8/9) and (4/15), respectively.

large Fourier components near $\omega=\omega_{I}$. The Zeeman-limit expression for $R_{1 p}$ is (taking $\omega_{I} \ll \omega_{S}$ ),

$$
\begin{aligned}
R_{1 p}=R_{1 z}+R_{1 \pm}= & \left(\frac{2}{15}\right) \frac{\left(\gamma_{I} g \beta_{o}\right)^{2}}{r^{6}}\left(\frac{\mu_{o}}{4 \pi}\right)^{2} S(S+1) \\
& \times\left[3 J_{z}\left(\omega_{I}\right)+7 J_{ \pm}\left(\omega_{S}\right)\right] .
\end{aligned}
$$

The first and second terms in brackets can be shown ${ }^{41}$ to result, respectively, from the interaction of $I$ with the dipolar fields of $\mu_{z}(t)$ and $\mu_{ \pm}(t)$. The corresponding dipolar power spectrum is shown in Fig. 4. The transition from the orthorhombic zfs limit to the Zeeman limit involves a shift in the dipolar power band associated with $\mu_{z}(t)$ from a center frequency of $\omega=2 \omega_{E}$ in the zfs limit, to a center frequency of $\omega=0$ in the Zeeman limit. This shift provides increased dipolar power at $\omega_{I}$ and produces, when $2 \omega_{E} \tau_{S, \hat{z}}>1$, a dramatic rise in $R_{1 p}$ as the magnetic field strength rises from the zfs limit to the Zeeman limit. The magnitude of the rise can be calculated rather simply from the limiting expressions [Eqs. (20) and (24)].

As a second example, we consider the dependence of $R_{1 p}$ on the correlation time $\tau_{S, \hat{z}}$, in the situation where $\tau_{S, \hat{z}}$ is long enough $\left(\tau_{S, \hat{z}}^{-1} \ll \omega_{D}\right)$ that overlap of the power peaks due to $\mu_{\hat{x}}(t)$ and $\mu_{\hat{y}}(t)$ with that due to $\mu_{\hat{z}}(t)$ is small at $\omega=0$. In this case, $R_{1 p} \cong R_{1 \hat{z}}$, and the power spectrum is qualitatively similar to that in Fig. 3(b). We have observed ${ }^{32}$ in spin dynamics simulations that $R_{1 p}$ is a monotonically increasing function of $\tau_{S, \hat{z}}$ when the zfs tensor is uniaxial, but it is often a decreasing function of $\tau_{S, \hat{z}}$ in the orthorhombic case. The spectral density representation provides a simple description of this behavior, as illustrated in Figs. 5 and 6. In the uniaxial zfs limit $\left(\omega_{E}=0\right)$, the dipolar power band associated with $\mu_{\bar{z}}(t)$ is centered at $\omega=0 . R_{1 p}$ is then proportional to $J_{\hat{z}}(0)$ and increases linearly with $\tau_{S, \hat{z}}$. When the $\mathrm{zfs}$ tensor is orthorhombic, $R_{1 p}$ is proportional to $J_{\hat{z}}\left(2 \omega_{E}\right)$, which passes through a maximum at $2 \omega_{E} \tau_{S, \hat{z}}=1$ (Fig. 6). When $\tau_{S, \hat{z}}>\left(2 \omega_{E}\right)^{-1}, J_{\hat{z}}(0)$ and $R_{1 p}$ decrease with increasing $\tau_{S, \hat{z}}$. 


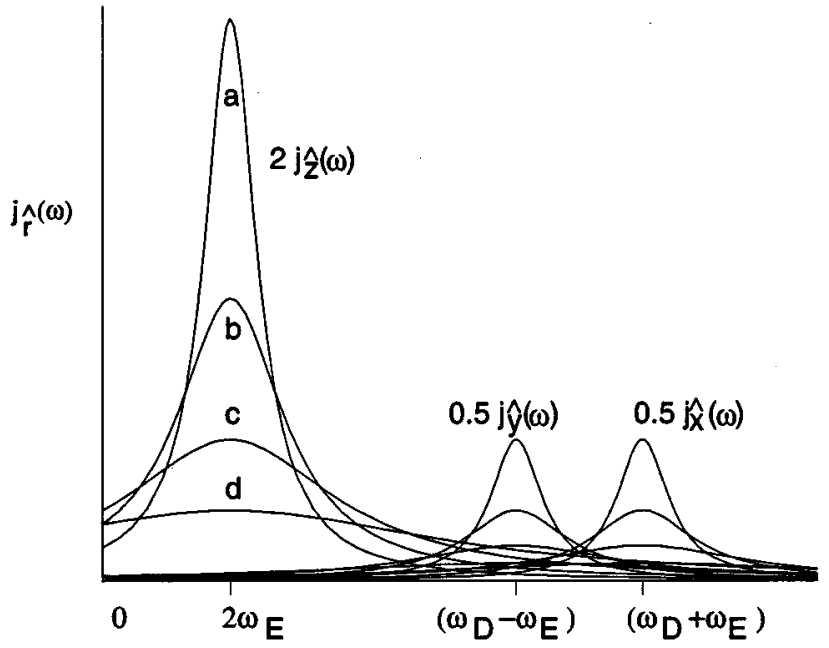

FIG. 5. Dependence of the dipolar power spectrum on the correlation time $\tau_{S, \hat{z}}$ in the orthorhombic zfs limit. Curves are shown for selected values of $\tau_{S, \hat{r}}$ equal to (a) $2 / \omega_{E}$, (b) $1 / \omega_{E}$, (c) $0.5 / \omega_{E}$, and (d) $0.25 / \omega_{E}$.

\section{ANALYSIS OF THE ZFS LIMIT AND INTERMEDIATE REGIME NMR-PRE DUE TO $\left[\mathrm{Ni}(\mathrm{II})(\mathrm{acac})_{2}\left(\mathrm{H}_{2} \mathrm{O}\right)_{2}\right]$}

Two of us (S.M.A. and R.R.S.) have previously reported ${ }^{34}$ measurements of the magnetic field dependence of the intermolecular $R_{1 p}$ of the dioxane solvent protons in solutions containing the $S=1$ complex [Ni(II) $\left.(\mathrm{acac})_{2}\left(\mathrm{H}_{2} \mathrm{O}\right)_{2}\right]$. These measurements confirmed the predicted increase in $R_{1 p}$ across the transition region between the zfs and Zeemanlimit regimes as described above. Quantitative analysis of the data was used in this study to place constraints on an acceptable range of $|E|$.

We report here measurements of the intramolecular proton $R_{1 p}$ for coordinated water protons in the axial positions

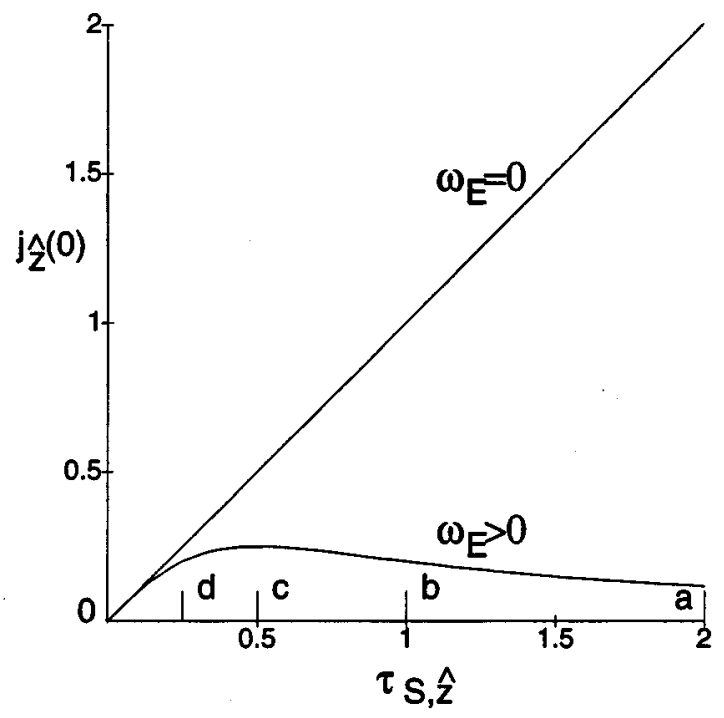

FIG. 6. Dependence of the dipolar power density at zero frequency on the electron spin relaxation time $\tau_{S, \hat{r}}$ in the uniaxial and orthorhombic zfs limits. Points (a) - (d) along the abscissa correspond to curves (a) - (d) in Fig. 5. $\tau_{S, \hat{z}}$ is units of $\omega_{E}^{-1}$.

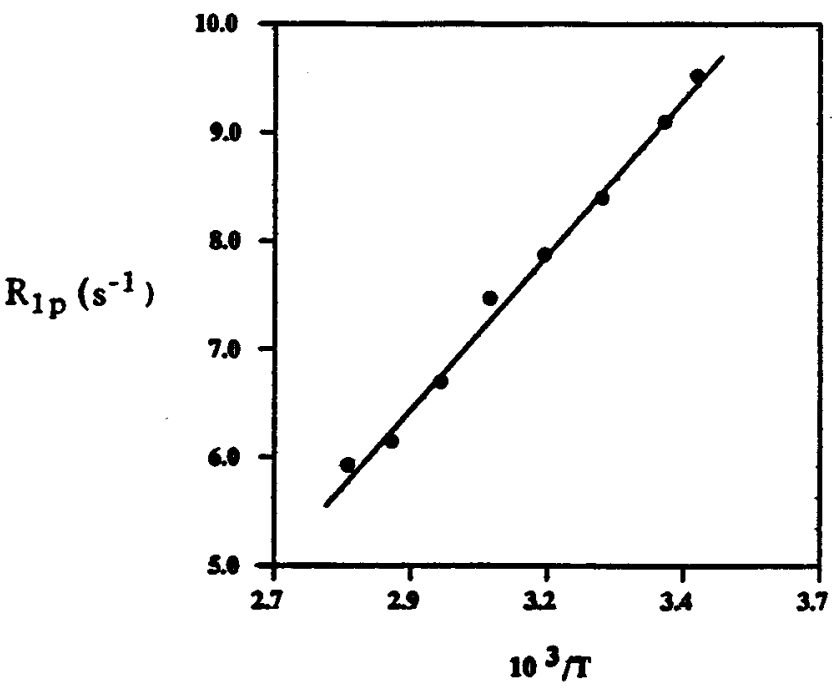

FIG. 7. Temperature dependence of the proton $T_{1}$ NMR relaxation enhancement $R_{1 p}$ for the water protons in [ $\left.\mathrm{Ni}(\mathrm{II})(\mathrm{acac})_{2}\left(\mathrm{H}_{2} \mathrm{O}\right)_{2}\right]$ in a mixed solvent containing $95 \%$ dioxane, $5 \%$ water $(\mathrm{v} / \mathrm{v})$. The proton resonance frequency is $20 \mathrm{MHz}$

of $\left[\mathrm{Ni}(\mathrm{II})(\mathrm{acac})_{2}\left(\mathrm{H}_{2} \mathrm{O}\right)_{2}\right]$. These measurements, which were made on a sample containing $40 \mathrm{mM}$ complex in deuterated dioxane solvent plus $5 \%(\mathrm{v} / \mathrm{v})$ water, permit an extension and refinement of our earlier analysis. They also illustrate the utility of the theory developed above as an aid for understanding and analyzing the physical information that is contained in NMR-PRE data. Experimental samples were used which contained excess unbound water that was in rapid chemical exchange equilibrium with axial positions in the complex. In the presence of rapid chemical exchange, the relaxation rate of the water proton resonance provides a measurement of the intramolecular $T_{1 m}$ (i.e., $T_{1}$ of the bound water) through the Luz-Meiboom relation, ${ }^{42}$

$$
R_{1 p}=\frac{f_{m}}{T_{1 m}+\tau_{m}} .
$$

$T_{1 m}$ and $\tau_{m}$ are the spin relaxation time and the mean chemical exchange lifetime of protons in the metal coordination sphere, and $f_{m}$ is the mole ratio of bound to unbound water in solution. We first confirmed that chemical exchange is rapid through variable temperature $R_{1 p}$ measurements, which are shown in Fig. 7. $R_{1 p}$ decreased with increasing temperature, confirming that $\tau_{m} \ll T_{1 m}$. The magnetic field dependence of $R_{1 m}$ of the bound water protons at $20{ }^{\circ} \mathrm{C}$ is shown in Fig. 8. The intramolecular $R_{1 p}$ data, like the intermolecular $R_{1 p}$ data, exhibited the predicted rise in $R_{1 p}$ upon passing from the zfs limit into the intermediate regime.

We first analyzed the zfs-limit $R_{1 p}$ data using Eq. (23). The intramolecular $R_{1 p}$ depends on two geometrical parameters, the interspin distance $r$ and the polar angle $\theta$ of the interspin vector in the zfs-PAS. Both of these values are known fairly accurately from the x-ray structure ${ }^{43}: r$ $=2.79 \AA$ and $\theta=16^{\circ}$. The reorientational correlation time $\tau_{R}^{(1)}$ for $\left[\mathrm{Ni}(\mathrm{II})(\mathrm{acac})_{2}\left(\mathrm{H}_{2} \mathrm{O}\right)_{2}\right]$ in dioxane at $20^{\circ} \mathrm{C}$ is $\tau_{R}^{(1)}=330 \mathrm{ps},{ }^{34}$ a value long enough that it has little influence 


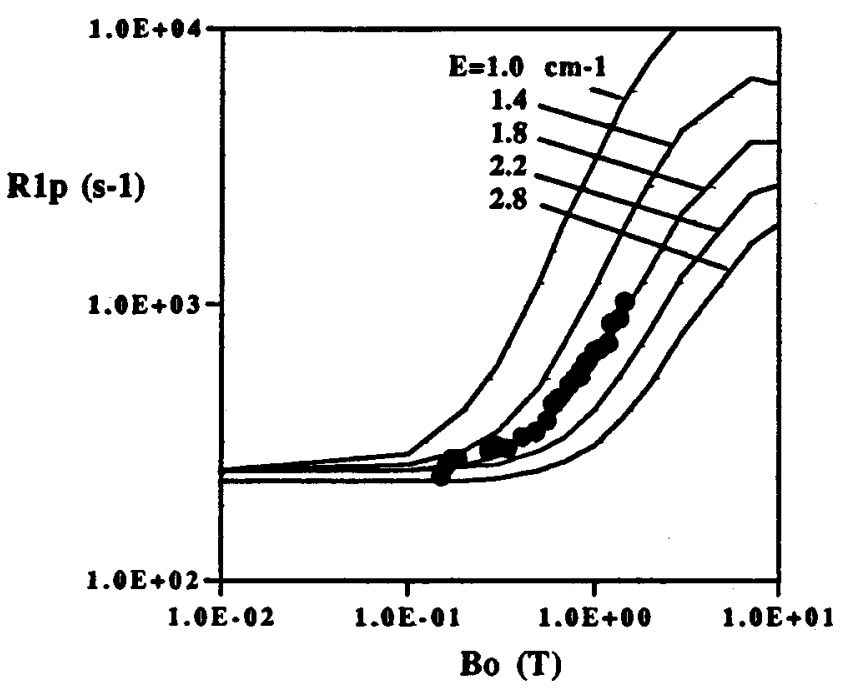

FIG. 8. Comparison of the results of SpinDyn.f simulations with $R_{1 p}$ field dispersion profile data (filled circles) at $20^{\circ} \mathrm{C}$. The SD simulations were performed assuming $|D|=10.0$ with $|E|=0.0,1.4,1.8,2.2$, and $2.8 \mathrm{~cm}^{-1}$, as indicated. The electron spin relaxation time $\tau_{S, \hat{z}}$ of each simulation was calculated as required to fit the low field data using a procedure described in the text.

on the analysis. According to Eqs. (23), the zfs-limit $R_{1 p}$ is proportional to the quantity $J=2.0 J_{\hat{z}}\left(2 \omega_{E}\right)+0.5\left[J_{\hat{x}}\left(\omega_{D}\right.\right.$ $\left.\left.+\omega_{E}\right)+J_{\hat{y}}\left(\omega_{D}-\omega_{E}\right)\right]$, where the numerical factors are the $\theta$-dependent quantities. From the experimental zfs-limit value of $R_{1 p}=240 \mathrm{~s}^{-1}$, we calculated $J=0.518 \times 10^{-12} \mathrm{~s}$.

Keeping this value fixed, we then performed spin dynamical simulations in which $|E|,|D|$, and $\tau_{S, \hat{r}}$ were permitted individually to vary (Fig. 8). The fitting procedure depended rather sensitively on $|E|$ and $\tau_{S, \hat{z}}$ but was nearly independent of $|D|, \tau_{S, \hat{x}}$, and $\tau_{S, \hat{y}}$. Satisfactory fits to the data required the values $|E|=1.8 \pm 0.1 \mathrm{~cm}^{-1}$ and $\tau_{S, \hat{z}}=7.9$ \pm 0.3 ps. The dependence of the analysis on only these two parameters is well explained in the spectral density representation. Figure 9 shows the three Cartesian spectral density functions $\left[1+P_{2}\left(\cos \theta_{\hat{r}}\right)\right] j_{\hat{r}}\left(\omega_{\hat{r}}\right),(\hat{r}=\hat{x}, \hat{y}, \hat{z})$, computed for

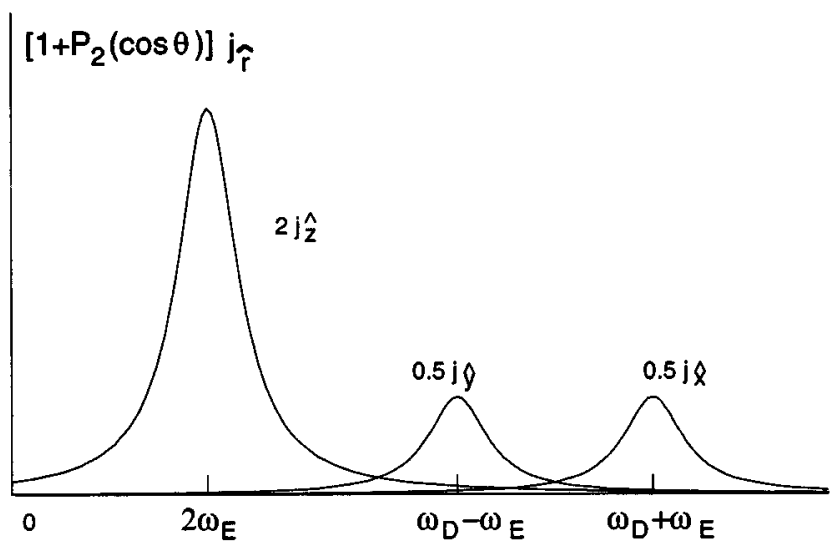

FIG. 9. Dipolar power density representation of the NMR relaxation enhancement of the water protons in $\left[\mathrm{Ni}(\mathrm{II})(\mathrm{acac})_{2}\left(\mathrm{H}_{2} \mathrm{O}\right)_{2}\right]$. Parameters were $|D|=10 \mathrm{~cm}^{-1},|E|=1.8 \mathrm{~cm}^{-1}, \tau_{S, \hat{r}}=8.0 \mathrm{ps}$.

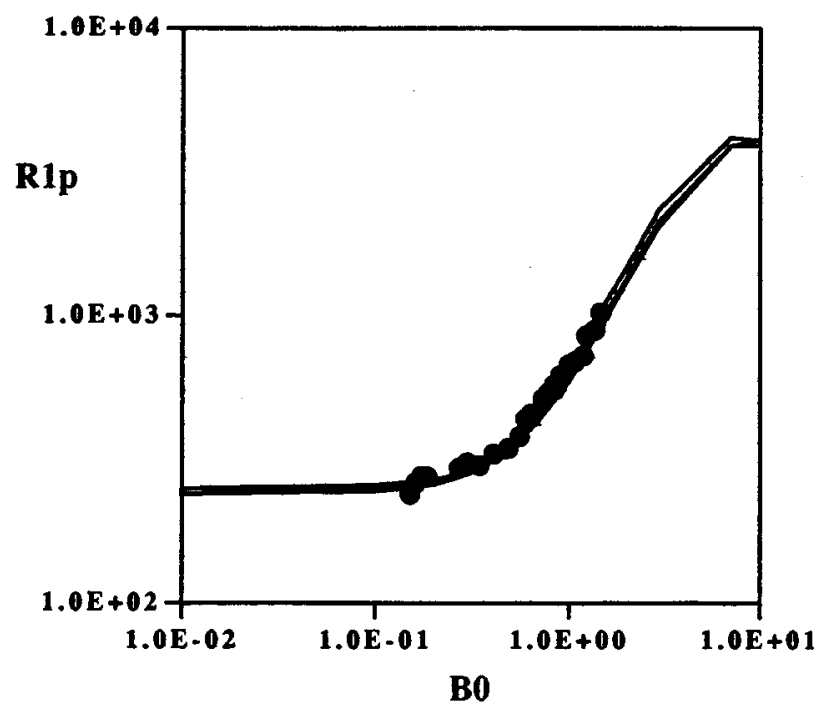

FIG. 10. Effect of variations of the zfs $D$-parameter on the spin dynamics simulations of $R_{1 p}$. Experimental data are solid circles. Simulated curves are shown for $D=7$ (upper), $D=10$ and $D=15 \mathrm{~cm}^{-1}$, with $E=1.8 \mathrm{~cm}^{-1}$ and $\tau_{S, \hat{z}}=8.0 \mathrm{ps}$. The curves for $D=10$ and $D=15 \mathrm{~cm}^{-1}$ coincide.

$|D|=10 \mathrm{~cm}^{-1}, \quad|E|=1.80 \mathrm{~cm}^{-1}$, and $\tau_{S, \hat{z}}=8.0 \mathrm{ps}$. These functions describe the dipolar power density due to $\mu_{\hat{x}}(t)$, $\mu_{\hat{y}}(t)$, and $\mu_{\hat{z}}(t)$. It is evident from the figure that the spectral contribution at $\omega_{I}$ due to $\mu_{\hat{z}}(t)$ is much greater than the contributions due to $\mu_{\hat{x}}(t)$ or $\mu_{\hat{y}}(t)$. For this reason, only the eigenfrequency and relaxation time of $\mu_{\hat{z}}(t)$, which are $2 \omega_{E}$ and $\tau_{S, \hat{z}}$, are significant parameters of the analysis. The simulations were virtually independent of $D$, as is shown in Fig. 10, which compares the results of three spin dynamics (SD) simulations performed using different values of $|D|$, $|D|=7,10$, and $15 \mathrm{~cm}^{-1}$, with $|E|$ and $\tau_{S, \hat{z}}$ fixed at $1.80 \mathrm{~cm}^{-1}$ and $8.0 \mathrm{ps}$. The calculated curves for $|D|=10$ and $15 \mathrm{~cm}^{-1}$ coincide within the width of the line, while the simulated curve for $|D|=7 \mathrm{~cm}^{-1}$ (upper curve) differs only slightly. Acceptable fits to the data were not found for $|D|$ $<5.5 \mathrm{~cm}^{-1}$. This constraint on $|D|$ does not represent a direct dependence of $R_{1 p}$ on $|D|$, but rather the tight constraint on $|E|$ in the analysis $\left(|E|=1.80 \mathrm{~cm}^{-1}\right)$, plus the fact that the maximum physical value of the $|E / D|$ ratio is $1 / 3$.

Spectral density representations based on Eqs. (18)-(20) provide a very useful framework for analyzing NMR-PRE data and for understanding the information that the relaxation data contain. The present analysis has shown that the water proton $R_{1 p}$ data for $\left[\mathrm{Ni}(\mathrm{II})(\mathrm{acac})_{2}\left(\mathrm{H}_{2} \mathrm{O}\right)_{2}\right]$ provide sensitive measurements of $|E|$ and $\tau_{S, \hat{z}}$, while being quite insensitive to the other parameters of theory. The spectral density representation shows why this is the case and illustrates graphically the physical conditions under which the neglect of $J_{\hat{y}}\left(\omega_{D}-\omega_{E}\right)$ and $J_{\hat{x}}\left(\omega_{D}+\omega_{E}\right)$ is justified. Based on the above analysis for $\left[\mathrm{Ni}(\mathrm{II})(\mathrm{acac})_{2}\left(\mathrm{H}_{2} \mathrm{O}\right)_{2}\right]$, it seems very likely that field-dependent NMR-PRE data of many other zfs-limit integer spin systems will likewise depend largely on $|E|$ and $\tau_{S, \hat{z}}$. The situation for half-integer spins $S \geqslant 3 / 2$ needs further investigation. 
In a previous analysis ${ }^{34}$ of the intermolecular solvent proton relaxation enhancements produced by $\left[\mathrm{Ni}(\mathrm{II})(\mathrm{acac})_{2}\left(\mathrm{H}_{2} \mathrm{O}\right)_{2}\right]$ in dioxane, we reported a somewhat lower range for $|E|, 0.6 \leqslant|E|<1.0 \mathrm{~cm}^{-1}$, than that reported here. The analysis of the intramolecular data described above is more accurate than that of the prior intermolecular relaxation study because the molecular geometry relevant to intramolecular dipolar coupling is much more accurately defined than that for intermolecular dipolar coupling. Currently available theory of intermolecular relaxation represents the solute as a hard sphere which excludes the diffusing solvent nuclei from penetrating an effective radius $d_{c}$ (which enters the theory as $d_{c}^{-3}$ ). The theory does not account for nonspherical shape of the solute, but rather parameterizes such effects within $d_{c}$. This parameterization is likely to be rather poor in the vicinity of the zfs limit, however, particularly when intra- and intermolecular data for the same complex are compared quantitatively. Since the zfs-limit $R_{1 p}$ results principally from $\mu_{\hat{z}}(t)$, the highest relaxation efficiency occurs at near-axial positions of the complex with relatively small $\theta$. A hard sphere calculation of the intermolecular $R_{1 p}$ for a solute such as $\left[\mathrm{Ni}(\mathrm{II})(\mathrm{acac})_{2}\left(\mathrm{H}_{2} \mathrm{O}\right)_{2}\right]$, which has a shorter distance of closest approach in axial than in equatorial directions, will tend to underestimate the true $R_{1 p}$. An accurate calculation for the intermolecular case requires that the translational time correlation functions of theory be evaluated using a molecular coordinate frame description of electron spin motion and a nonspherical excluded volume to describe the solute molecular shape. We are currently undertaking calculations of this kind.

\section{SUMMARY AND CONCLUSIONS}

Molecular-frame theoretical expressions have been derived to describe the NMR relaxation enhancement produced by spin $S \geqslant 1$ transition metal ions in the orthorhombic zfs limit. These expressions, which are summarized in Eqs. (18a), (18b), and (19) for arbitrary $S$, and in Eq. (23) for $S$ $=1$, suggest a very simple physical interpretation of the dipolar relaxation mechanism. The nuclear spin relaxation enhancement $R_{1 p}$ is induced by the time-dependent local dipolar field associated with the electronic magnetic moment of $S$. The molecular-frame Cartesian components $\bar{\mu}_{\hat{r}}(t)$ of $\bar{\mu}(t)$ contribute additively to $R_{1 p}$. The dependence on the positional coordinates of the nuclear spin in the molecular coordinate frame are described by the functions $r^{-6}[1$ $+P_{2}\left(\cos \theta_{r}\right)$ ], where $r$ is the interspin distance and $\cos \theta_{\hat{r}}$ is the direction cosine of the interspin vector with the $\hat{\mathbf{r}}$ th coordinate axis of the zfs-PAS. These functions describe the geometric dependence of the squared local magnetic field that is produced by a magnetic dipole oriented along the $\hat{\mathbf{r}}$ th Cartesian axis. Each component $R_{1 \hat{r}}$ is proportional also to the dipolar power density produced by $\bar{\mu}_{\hat{r}}(t)$ at the nuclear Larmor precession frequency $\omega_{I}$. Dipolar power representations are shown to provide a physically transparent description of the spin relaxation mechanism. These representations provide a simple way of understanding the physical informa- tion that is contained in the experimental data and of differentiating between the sensitive and insensitive parameters of theory.

The molecular-frame theoretical expressions presented permit the inclusion, in a straightforward way, of Cartesian parameters to represent the effects of molecular anisotropies of electron spin relaxation and of Brownian reorientational diffusion. Molecular anisotropy of the former kind, i.e., different electron spin relaxation rates along different molecular axes, is likely to have very important experimental consequences in complexes where the electronic environment of the metal ion is far from cubic, as, for example, in metalporphyrins. A molecular-frame Redfield-type theory of electron spin relaxation is needed to describe these effects.

\section{EXPERIMENT}

$\left[\mathrm{Ni}(\mathrm{II})(\mathrm{acac})_{2}\left(\mathrm{H}_{2} \mathrm{O}\right)_{2}\right]$ was synthesized and characterized as described previously. ${ }^{34}$ A $20 \mathrm{mM}$ solution of the complex was prepared in a mixed solvent containing $5 \%(\mathrm{v} / \mathrm{v})$ water in dioxane- $\mathrm{d}_{8} .200 \mu \mathrm{l}$ of this solution was transferred to a 10 $\mathrm{mm}$ glass tube which had been washed in concentrated sulfuric acid and rinsed with distilled, deionized water to remove labile paramagnetic ions on the surface. The sample was degassed by four freeze-pump-thaw cycles and sealed under vacuum. The $T_{1}$ measurement techniques are described in Ref. 34.

\section{ACKNOWLEDGMENTS}

R.R.S. and S.M.A. acknowledge financial support by the U.S. National Science Foundation in the form of a research grant, CHE-9423351. S.M.A. received financial support in the form of a Rackham Merit Fellowship from the University of Michigan.

${ }^{1}$ I. Solomon, Phys. Rev. 99, 559 (1955).

${ }^{2}$ N. Bloembergen, J. Chem. Phys. 27, 572 (1957); 27, 595 (1957).

${ }^{3}$ N. Bloembergen and L. O. Morgan, J. Chem. Phys. 34, 842 (1961).

${ }^{4}$ A. Abragam and B. Bleaney, Electron Paramagnetic Resonance of Transition Ions (Dover, New York, 1970).

${ }^{5}$ L. L. Lohr, Mol. Phys. 89, 1397 (1996).

${ }^{6}$ H. L. Friedman, M. Holz, and H. G. Hertz, J. Chem. Phys. 70, 3369 (1969)

${ }^{7}$ J. H. Freed, G. V. Bruno, and C. Polnaszek, J. Chem. Phys. 55, 5270 (1971); 56, 716 (1972).

${ }^{8}$ N. Benetis, J. Kowalewski, L. Nordenskiold, H. Wennerstrom, and P.-O. Westlund, Mol. Phys. 48, 329 (1983).

${ }^{9}$ N. Benetis, J. Kowalewski, L. Nordenskiold, H. Wennerstrom, and P.-O. Westlund, J. Magn. Reson. 58, 261 (1984).

${ }^{10}$ P.-O. Westlund, H. Wennerstrom, L. Nordenskiold, J. Kowalewski, and N. Benetis, J. Magn. Reson. 59, 91 (1984).

${ }^{11}$ N. Benetis, J. Kowalewski, L. Nordenskiold, and U. Edlund, J. Magn. Reson. 58, 282 (1984).

${ }^{12}$ I. Bertini, C. Luchinat, M. Mancini, and G. Spina, J. Magn. Reson. 59, 213 (1984).

${ }^{13}$ T.-H. R. Chen, S.-J. Den, and L.-P. Hwang, Proc. Natl. Sci. Council (Taiwan) 8A, 224 (1984).

${ }^{14}$ L.-P. Hwang and C.-Y. Ju, J. Chem. Phys. 83, 3775 (1985).

${ }^{15}$ I. Bertini, C. Luchinat, and J. Kowalewski, J. Magn. Reson. 62, 235 (1985).

${ }^{16}$ N. Benetis and J. Kowalewski, J. Magn. Reson. 65, 13 (1985). 
${ }^{17}$ L. Banci, I. Bertini, F. Briganti, and C. Luchinat, J. Magn. Reson. 66, 58 (1986).

${ }^{18}$ P.-L. Wang, J.-H. Lee, S.-M. Huang, and L.-P. Hwang, J. Magn. Reson. 73, 277 (1987)

${ }^{19}$ R. R. Sharp, J. Chem. Phys. 93, 6921 (1990).

${ }^{20}$ T. Bayburt and R. R. Sharp, J. Chem. Phys. 92, 5892 (1990).

${ }^{21}$ H. Fukui, K. Miura, and H. Matsuda, J. Magn. Reson. 88, 311 (1990).

${ }^{22}$ R. R. Sharp, J. Magn. Reson. 100, 491 (1992).

${ }^{23}$ T. Bayburt and R. R. Sharp, J. Phys. Chem. 97, 4558 (1993).

${ }^{24}$ R. R. Sharp, J. Chem. Phys. 98, 912 (1993).

${ }^{25}$ R. R. Sharp, J. Chem. Phys. 98, 2507 (1993)

${ }^{26}$ J.-M. Bovet and R. R. Sharp, J. Chem. Phys. 99, 18 (1993).

${ }^{27}$ T. Larsson, P.-O. Westlund, J. Kowalewski, and S. H. Koenig, J. Chem. Phys. 101, 1116 (1994).

${ }^{28}$ I. Bertini, O. Galas, C. Luchinat, and G. Parigi, J. Magn. Reson. A 113, 151 (1995)

${ }^{29}$ M. Odelius, C. Ribbing, and J. Kowalewski, J. Chem. Phys. 103, 1800 (1995).

${ }^{30}$ M. Odelius, C. Ribbing, and J. Kowalewski, J. Chem. Phys. 104, 3181 (1996).
${ }^{31}$ J. Svoboda, T. Nilsson, J. Kowalewski, P.-O. Westlund, and P. T. Larsson, J. Magn. Reson. 121, 108 (1996).

${ }^{32}$ P.-O. Westlund, in Dynamics of Solutions and Fluid Mixtures by NMR, edited by J. J. Delpuech (Wiley, New York, 1995), p. 173.

${ }^{33}$ S. M. Abernathy and R. R. Sharp, J. Chem. Phys. 106, 9032 (1997).

${ }^{34}$ S. M. Abernathy and R. R. Sharp, J. Phys. Chem. 202, 3692 (1997).

${ }^{35}$ R. A. Dwek, NMR in Biochemistry (Oxford University Press, Oxford, 1972).

${ }^{36}$ C.-W. Chen, J. S. Cohen, C. E. Myers, and M. Sohn, FEBS Lett. 168, 70 (1984).

${ }^{37}$ S. H. Koenig, R. D. Brown, and M. Spillar, Magn. Reson. Med. 4, 252 (1987)

${ }^{38}$ K. E. Keller and N. Foster, Inorg. Chem. 31, 1353 (1992).

${ }^{39}$ R. Kubo and K. Tomita, J. Phys. Soc. Jpn. 9, 888 (1954).

${ }^{40}$ L. Banci, I. Bertini, and C. Luchinat, Nuclear and Electron Relaxation (VCH, New York, 1991), pp. 185-191.

${ }^{41}$ S. H. Koenig, J. Magn. Reson. 31, 1 (1978).

${ }^{42}$ Z. Luz and S. Meiboom, J. Chem. Phys. 40, 2686 (1964).

${ }^{43}$ H. Montgomery and E. C. Lingafelter, Acta Crystallogr. 17, 1481 (1964). 